\title{
Klebsiella Pneumoniae-related Brain Abscess and Meningitis in Adults: Case Report and Literature Review
}

\section{Jingru Zhao}

Hebei General Hospital

Tiantian Huo

Hebei General Hospital

\section{Xintong Luo}

Hebei General Hospital

\section{Litao Li}

Hebei General Hospital

Baoming Yang ( $\nabla$ ybm0908@163.com )

Hebei Medical University Fourth Affiliated Hospital and Hebei Provincial Tumor Hospital https://orcid.org/0000-0003-3609-1461

\section{Case report}

Keywords: Klebsiella, brain abscess, meningitis

Posted Date: June 16th, 2021

DOl: https://doi.org/10.21203/rs.3.rs-591081/v1

License: (c) (1) This work is licensed under a Creative Commons Attribution 4.0 International License. Read Full License 


\section{Abstract}

Background: Despite the progress of modern neurosurgical techniques, new antibiotics and modern imaging techniques, brain abscess is still a potentially fatal infection. Streptococci have always been the common organisms resulting in brain abscess. Nevertheless, Klebsiella species, once thought to be a less common cause of brain abscess in adults, have become an increasingly important cause of brain abscess, especially in Asia.

Case presentation: A 64-year-old woman felt sudden onset severe continuous headache, accompanied by intermittent nausea, vomiting and fever. Meanwhile, she felt tinnitus and had a feeling of swelling in the right ear. Electroencephalogram (EEG) examination showed mild abnormality. A neurological examination revealed obvious nuchal rigidity with four transverse fingers under the chin. Cranial magnetic resonance imaging (MRI) revealed abnormal hyperintensities signals in the left head of caudate nucleus. The cerebral spinal fluid (CSF) common results indicated bacterial infection The next generation sequencing (NGS) of CSF showed the infection of K pneumoniae. The patient was diagnosed with Klebsiella pneumoniae-related brain abscess and meningitis. After positive antimicrobial treatment for nearly two months. The patient got a good recovery.

Conclusion: $\mathrm{K}$ pneumoniae is once thought to be a less common cause of brain abscess in adults and mainly hospital-acquired. Community-acquired CNS infection (brain abscess and meningitis) caused by $\mathrm{K}$ pneumoniae without other metastatic septic abscesses is exceedingly rare. Therefore, we present a rare adult patient with invasive cerebral abscess and meningitis without other invasive abscesses related to $\mathrm{K}$ pneumoniae.

\section{Background}

Despite the progress of modern neurosurgical techniques, new antibiotics and modern imaging techniques, brain abscess is still a potentially fatal infection caused primarily by spread from infected parameningeal or remote foci [1]. Streptococci have always been the common organisms resulting in brain abscess. Nevertheless, Klebsiella species, once thought to be a less common cause of brain abscess in adults, have become an increasingly important cause of brain abscess in Asia [2]. $\mathrm{K}$ pneumoniae is mainly hospital-acquired [3]. Community-acquired CNS infection (brain abscess and meningitis) caused by $\mathrm{K}$ pneumoniae without other metastatic septic abscesses is exceedingly rare. Therefore, we present a rare adult patient with invasive cerebral abscess and meningitis without other invasive abscesses related to $\mathrm{K}$ pneumoniae.

\section{Case Presentation}

A 64-year-old woman felt sudden onset severe continuous headache, especially in the front temporal occipitoparietal areas, accompanied by intermittent nausea and vomiting on 21 st June, 2020. Meanwhile, she felt tinnitus and had a feeling of swelling in the right ear, whereas, without fever, lateral limb 
weakness, blurred vision and loss of consciousness. Electroencephalogram (EEG) examination showed mild abnormality. Upon being admitted on 26th June, the patient developed a fever with the highest point reaching $39.5^{\circ} \mathrm{C}$. A neurological examination revealed obvious nuchal rigidity with four transverse fingers under the chin. However, he had intact sensation and full limb power with a negative bilateral Babinski sign.

Cranial magnetic resonance imaging (MRI) revealed abnormal hyperintensities signals in the left head of caudate nucleus, with T1-weighted sequence of hypointensity, T2 -weighted sequence of hyperintensity, FLAIR sequence of slightly hyperintensity, and diffusion restriction on diffusion-weighted imaging (DWI) and apparent diffusion coefficient (ADC) at the corresponding position. No significant abnormality was observed in the cerebral magnetic resonance angiography (MRA). Therefore, we further conducted cranial SPGR and meningeal CUBE enhancement, which displayed the ring-enhancement with consistent thickness next to the anterior horn of the left lateral ventricle and the meningeal linear enhancement in the left occipital lobe, suggesting a high probability of infectious diseases of brain abscess and meningitis (Fig. 1A-1 J).

Blood analysis showed elevated levels of neutrophile granulocyte percentage (84.6\%), C-reactive protein (CRP) $(238.88 \mathrm{mg} / \mathrm{L})$, erythrocyte sedimentation rate (ESR) $(82 \mathrm{~mm} / \mathrm{h})$, and procalcitonin (HCT) $(0.228 \mathrm{ng} / \mathrm{ml})$. Accordingly, we gave the patient anti-infective therapy of ceftriaxone $(2 \mathrm{~g} \mathrm{Q12h})$ and metronidazole $(0.5 \mathrm{~g} \mathrm{Q8h})$ by experience. The temperature began to go down, fluctuating between $37.0^{\circ} \mathrm{C}$ and $37.8^{\circ} \mathrm{C}$. The antinuclear antibody, hepatitis and tumor screening, coagulation test, thyroid function, vasculitis screening, brucella agglutination test, tiger red plate agglutination test, Brucella IgG antibody detection, Widal and Weil-Felix reaction, anti-human globulin test, T cell test for tuberculous infection, HIV, TP, blood culture, cardiac ultrasound, chest CT, cranial CT and abdomen CT showed no obvious abnormality. The CT scan of temporal bone and paranasal sinuses, which showed right otitis mastoidea (Fig. $1 \mathrm{~K}, 1 \mathrm{~L}$ ). The otolaryngology consultation considered the right otitis media, and $0.2 \mathrm{ml}$ of sallow exudate was extracted.

The cerebral spinal fluid (CSF) common results on 29th June were shown in Table 1 and Fig. 2. The CSF cultures, acid-fast dyeing, and India ink staining were negative. The next generation sequencing (NGS) of CSF showed the infection of $\mathrm{K}$ pneumoniae. Ceftriaxone and metronidazole were replaced by meropenem to broaden the antimicrobial spectrum, and $20 \%$ mannitol ( $125 \mathrm{ml} \mathrm{Q} 8 \mathrm{~h}$ ) was added to reduce the intracranial pressure. The patient's body temperature dropped below $37.3^{\circ} \mathrm{C}$ and the symptom of headache and fever alleviated obviously. The CSF results got better on 6th July (Table 1, Fig. 2). The patient asked for discharge. The cranial SPGR and meningeal CUBE enhancement on 10th July displayed the abscess cavity and wall was larger and thicker than before (Fig. 3A-3D). The patient went back to the local hospital and gave the ceftriaxone injection for 8 weeks. The CSF results got better on 22th July (Table 1, Fig. 2). Almost two months after the onset (31st August), the CSF results returned nearly to normal (Table 1, Fig. 2), and the lesions in MRI nearly disappeared (Fig. 3E-3J). She didn't complain of anything uncomfortable until 28th December. 


\section{Discussion}

Purulent meningitis is often combined with purulent encephalitis or brain abscess. Though further MRI image, CSF analysis, NGS results, the therapeutic effects, we confirmed that this patient was accompanied with $\mathrm{K}$ pneumoniae-associated solitary brain abscess secondary to meningitis, which was relatively rare in CNS infection in adults without history of trauma, surgery and cerebrospinal fluid leakage.

$\mathrm{K}$ pneumoniae, a gram-negative, Enterobacteriaceae, is mainly hospital-acquired and inclined to results in multiple abscess in patients with fragile immune system [4]. Nevertheless, community-acquired K. pneumoniae related solitary brain abscess without other metastatic septic abscesses is rare. The patient in our case seldomly went to hospital before being hospitalized this time, and without other medical history except chronic otitis media, suggesting us the high probability of community-acquired $\mathrm{K}$ pneumoniae focal CNS without invasion to other organs, which is very rare and is in accordance with previous rare report [5]. Accordingly, it gave us a reminder that $\mathrm{K}$ pneumoniae could not be completely excluded in the CNS community-acquired infection, especially in Asia. The NGS is still necessary in guiding diagnosis and treatment of CNS infectious diseases.

Brain abscess usually results from predisposing factors, such as otitis media, mastoiditis, trauma, postneurosurgery, human immunodeficiency virus (HIV), long-term treatment with immunosuppressive drugs. Brain abscess from chronic otitis media and mastoiditis are usually caused by streptococcus species, and account for about half of cases. However, it is notable that enterobacteriaceae could not be ignored in recent years. In this patient, based on the history, the sense of ear swelling, temporal bone CT, the excretion from the ear, negative blood culture, and the solitary brain abscess without multiple invasions, we speculated the infectious sources might be related to middle ear and mastoids. Nevertheless, it was a pity that we had not obtain the bacterial culture of the yellow exudate exacted from the ear, which might be a possible way to prove the brain abscess sources. Accordingly, we are supposed to try the best to perfect relevant inspection to specify the pathogenesis in future clinical work.

Empirical anti-infective treatment for patients with contiguous spread from a parameningeal infection and no history of neurosurgery consists of ceftriaxone/cefotaxime combined with metronidazole, or meropenem. Cefotaxime or ceftriaxone is recommended for the treatment based on isolated pathogen for enterobacteriaceae. The duration of intravenous antimicrobial therapy in patients with bacterial brain abscess has traditionally been 6 to 8 weeks [6]. Important criteria for evaluating treatment are the neurologic condition of the patient and abscess size on image examination. In this patient, ceftriaxone combined with metronidazole, and meropenem were all used to cover anaerobes and broaden antibacterial spectrum in the early stage, and ultimately ceftriaxone used for nearly two months got the good therapeutic effects.

In summary, if a bacterial CNS infection is strongly suspected but the culture results are negative, NGS may provide a definitive etiologic diagnosis. When brain abscess occurs in patients with otitis media and mastoiditis, immune-deficiency patients or trauma patients, bacteriaceae infection should not be ignored 
in addition to the common streptococci infection. If the clinical doctors make the diagnosis of $\mathrm{K}$ pneumoniae CNS infection, they are supposed to consider the characteristic of migration infection and make sure whether other metastatic septic abscesses exist or not. Early, regular and long-term antibiotics therapy are important for the patient's favorable clinical outcomes.

\section{Declarations}

\section{Ethics approval and consent to participate}

According to local ethical regulations (Ethics committee of the Heinrich Heine University, Düsseldorf, Germany) "case reports are not prospectively planned research projects on or with people, but retrospective case descriptions of medical actions. Therefore, the ethics committee is not responsible for evaluating case reports" and consequently is waiving the necessity of an ethical approval for case reports.

\section{Consent for publication}

Written informed consent for publication was obtained from the patient.

\section{Availability of data and materials}

The data generated during and/or analyzed during the current case report are available from the corresponding author on reasonable request.

\section{Competing interests}

No competing interests or conflicts exist for any author to declare.

\section{Funding}

No funding was received for this work.

\section{Authors' contributions}

All coauthors contributed equally to this work. All authors read and approved the final manuscript.

\section{Acknowledgements}

The authors would like to thank Shuqian Zhang and Jianhua Wang for their assistance in writing the manuscript.

\section{References}

1. Martin RM, Bachman MA. Colonization, Infection, and the Accessory Genome of Klebsiella pneumoniae. Front Cell Infect Microbiol. 2018; 8: 4. 
2. Liliang PC, Lin YC, Su TM, Rau CS, Lu CH, Chang WN, Lee TC, Chen HJ. Klebsiella brain abscess in adults. Infection. 2001; 29(2):81-86.

3. Calfee DP. Recent advances in the understanding and management of Klebsiella pneumoniae. F1000Research. 2017; 6: 1760.

4. Wang B, Zhang P, Li Y, Wang Y. Klebsiella pneumoniae-induced multiple invasive abscesses: A case report and literature review. Medicine. 2019; 98(39): e17362.

5. Sun R, Zhang H, Xu Y, Zhu H, Yu X, Xu J. Community-acquired Klebsiella pneumoniae central nervous system infection after acute suppurative otitis. IDCases 23. 2020; 23: e01016.

6. Brouwer MC, Tunkel AR, McKhann GM, van de Beek D. Brain abscess. The New England journal of medicine. 2014; 371(5): 447-456.

\section{Table}

Table 1 CSF results

\begin{tabular}{lcccc}
\hline CSF data & $2020-6-29$ & $2020-7-6$ & $2020-7-22$ & $2020-8-31$ \\
\hline CSF appearance & yellow & light yellow & transparent & transparent \\
CSF pressure $\left(\mathrm{mmH}_{2} \mathrm{O}\right)$ & 200 & 160 & 150 & 130 \\
Protein $(\mathrm{mg} / \mathrm{dL})$ & 74.49 & 73.98 & 31.43 & 28.65 \\
Glucose $1(\mathrm{mg} / \mathrm{dL})$ & 70.92 & 45.98 & 51.98 & 64.84 \\
Chlorine $(\mathrm{mmol} / \mathrm{L})$ & 120 & 117 & 128 & 129 \\
Leukocyte count $\left(\times 10^{6} / \mathrm{L}\right)$ & 400 & 232 & 57 & 6 \\
Percentage of neutrophils $(\%)$ & 59 & 30 & 1 & 1 \\
Percentage of lymphocyte(\%) & 30 & 61 & 84 & 95 \\
\hline
\end{tabular}

\section{Figures}



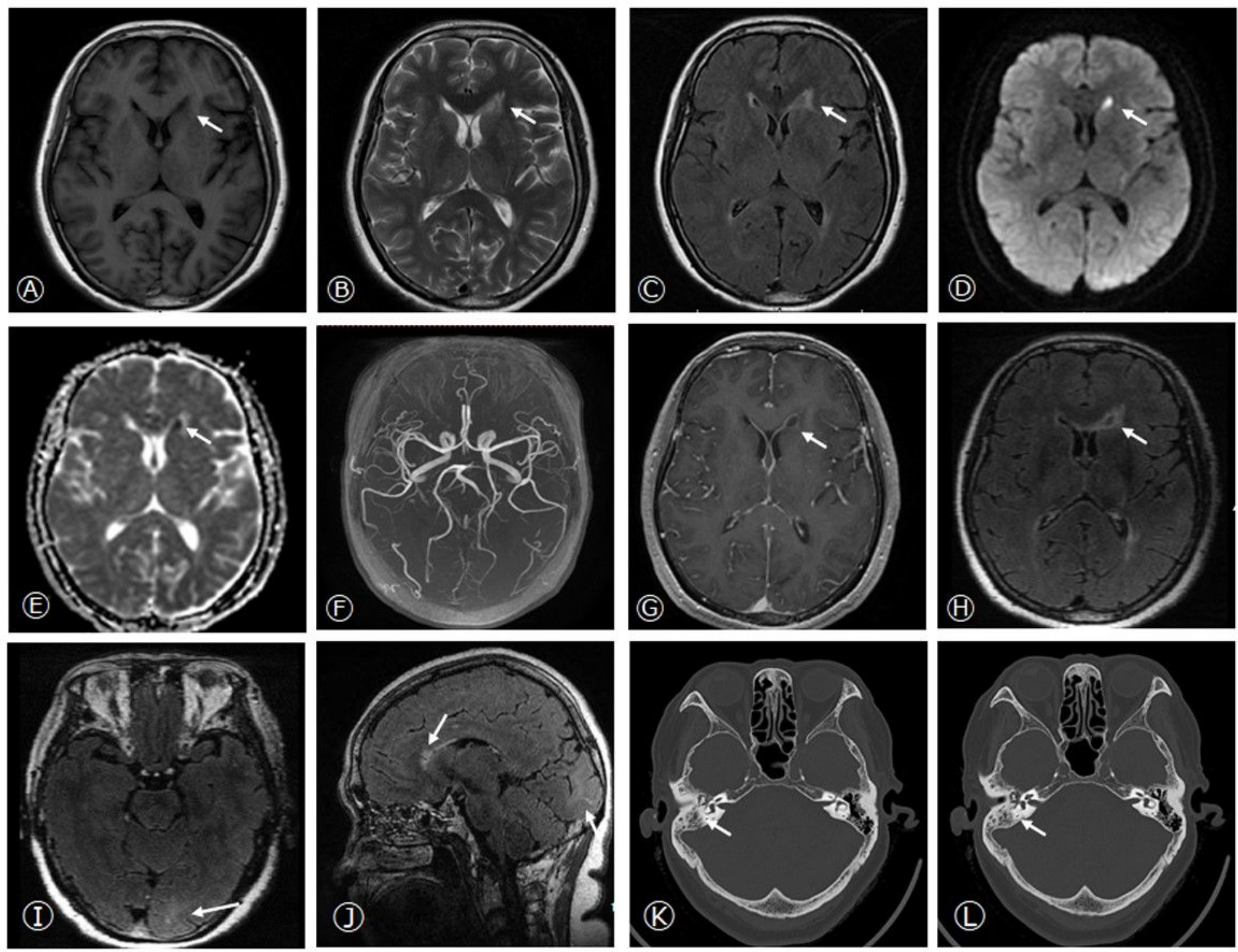

Figure 1

Brain MRI and Temporal bone CT at admission. Axial T1-weighted sequence, T2 -weighted sequence, FLAIR sequence (A-C), DWI (D), and ADC (E) sequences were imaged and showed abnormal signals in the left head of caudate nucleus, next to the anterior horn of lateral ventricle. No significant abnormality was observed in the cerebral MRA (F). Cranial SPGR and meningeal CUBE enhancement displayed the ringenhancement in the left head of caudate nucleus and meningeal linear enhancement in left occipital lobe on axial (G-I) and sagittal images (J). The temporal bone CT showed the right otitis mastoidea $(K, L)$.
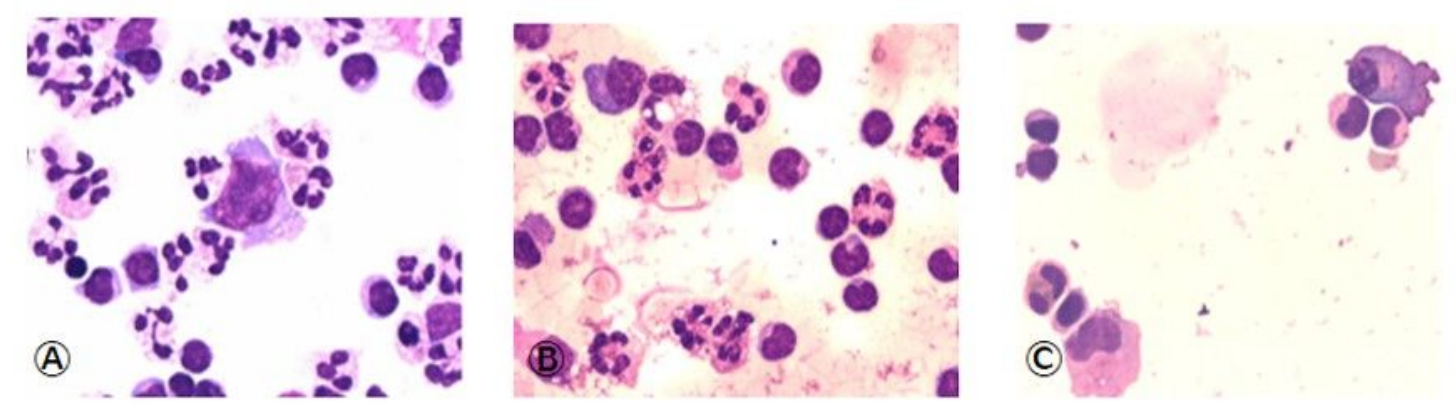
Figure 2

CSF cytology results on 29th June (A) 87 th July (B) $₫ 22$ nd July (C), and 31st August (D), respectively.
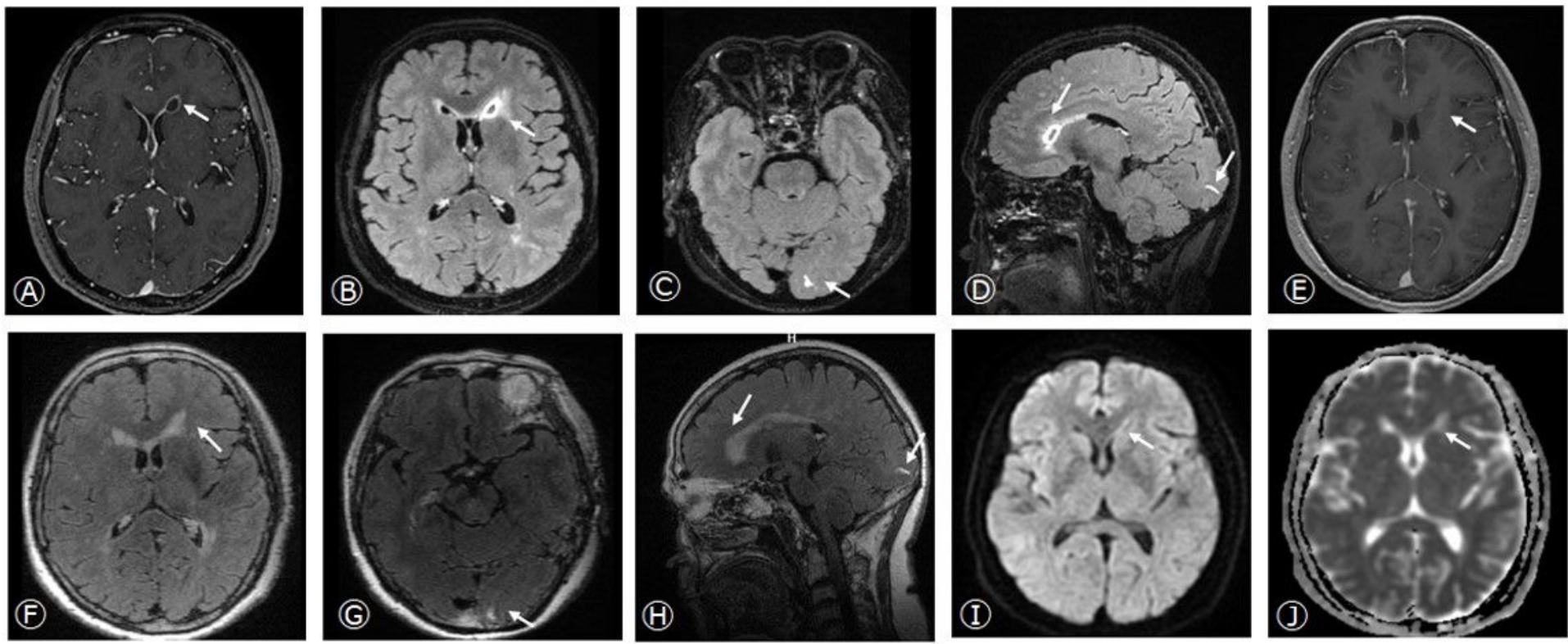

Figure 3

Brain MRI after therapy. Cranial SPGR (A) and meningeal CUBE enhancement (B-D) displayed the ringenhancement in the left head of caudate nucleus and linear enhancement in left occipital lobe on axial and sagittal images on 10th July, 2020. The cranial SPGR (E), meningeal CUBE enhancement (F-H), DWI $(\mathrm{I})$ and $\mathrm{ADC}(\mathrm{J})$ revealed nearly disappeared ring and linear enhancement on 31st August, 2020. 